\title{
The glial Gomori-positive material is sulfane sulfur
}

\author{
Zbigniew Srebro $^{1}$, Małgorzata Iciek ${ }^{2}$, Piotr Sura ${ }^{1}$, Marta Góralska ${ }^{1}$ \\ ${ }^{1}$ Department of Human Developmental Biology and ${ }^{2}$ Chair of Medical Biochemistry, \\ Medical College, Jagiellonian University, Kraków, Poland
}

\begin{abstract}
The Gomori-positive glia are periventricular astrocytes with abundant cytoplasmic granular material, predominantly occupying a periventricular site in the brain. These granular inclusions are strongly stained with chrome hematoxylin in the Gomori's method as well as exhibit red autofluorescence and non-enzymatic peroxidase activity. The glial Gomoripositive material (GGPM) granules are positive in the performic acid Alcian blue method indicating the presence of proteinbound sulfur, what has been shown by our previous studies. The number of cells containing glial Gomori-positive granules dropped after administration of cyanide and increased under the influence of sulfane sulfur donor (diallyl disulfide). This suggests, that sulfur of these granules is a sulfane sulfur, possibly in the form of protein-bound cysteine persulfide. Sulfane sulfur is labile, reactive sulfur atom covalently bound to another sulfur atom. In this paper we present evidence that GGPM exhibit affinity to cyanolysis and its stainability in Gomori's method is due to the presence of protein-bound sulfane sulfur. The biological role of the Gomori-positive glia connected with protective properties of sulfane sulfur has been discussed.
\end{abstract}

Key words: Glial Gomori-positive material - GGPM - Sulfane sulfur - Cyanolysis

\section{Introduction}

Cysteine may undergo oxidative metabolism leading to sulfates and taurine [1] or non-oxidative desulfuration that produces compounds containing sulfane sulfur atoms [2]. Sulfane sulfur is labile, highly reactive sulfur atom covalently bound to another sulfur atom and having oxidation state 0 or -1 . Sulfur with such features easily leaves structure of the compound and readily reacts with various acceptors, like thiols ( $\mathrm{RSH})$ or cyanide $(\mathrm{CN})[2,3]$. For the latter acceptor, such sulfur is also often called cyano-lysable sulfur [4].

Proteins, that transport reactive sulfur from anionic donors to thiophilic acceptors are rhodanese, (thiosulfate sulfurtransferase, EC 2.8.1.1.), 3-mercaptopyruvate sulfurtransferase (MPST, EC 2.8.1.2) and cystathionase (cystathionine $\gamma$-lyase, EC 4.4.1.1) as well as albumin $[2,5]$.

Sulfane sulfur fulfills regulatory functions, plays an important role in cyanide detoxification as well as participates in the formation of iron-sulfur proteins [2]. Regulatory properties of sulfane sulfur are related to the pos-

Correspondence: Piotr Sura, Department of Human Developmental Biology, Medical College, Jagiellonian University, Kopernika 7, 31-034 Kraków, Poland; tel./fax.: (+4812) 4229949, e-mail: mbsura@cyf-kr.edu.pl sibility of covalent modification of -SH groups of proteins with persulfide or trisulfide formation, which can directly influence their biological activity [5]. Good candidates as acceptors of sulfane sulfur are thioredoxin and metallothionein. Both proteins are rich in sulfhydryl groups and reduced thioredoxin is known to be a sulfuracceptor substrate for rhodanese [6]. In mammals, the activity of rhodanese and the closely related 3-mercaptopyruvate sulfurtransferase were found mainly in mitochondria $[7,8]$.

The Gomori-positive glia are a kind of astrocytes in hypothalamus, predominantly occupying a periventricular site in the brain and showing abundant cytoplasmic granular material. These granular inclusions exhibit an affinity for Gomori stains (chrome alum hematoxylin and aldehyde fuchsin) as well as red autofluorescence and non-enzymatic peroxidase activity [9-13]. In the preparations of astrocyte cytoplasmic granules from intact rat brain the smaller granules (0.5-4.0 microns) and larger inclusions (5-10 microns) were observed. The smaller granules were spherical and weakly autofluorescent, whereas larger inclusions tended to be intensely autofluorescent and pleomorfic [14]. The role of Gomori-positive astrocytes under normal and pathologic conditions is incompletely understood. Some studies show, that cysteamine exposure rapidly 

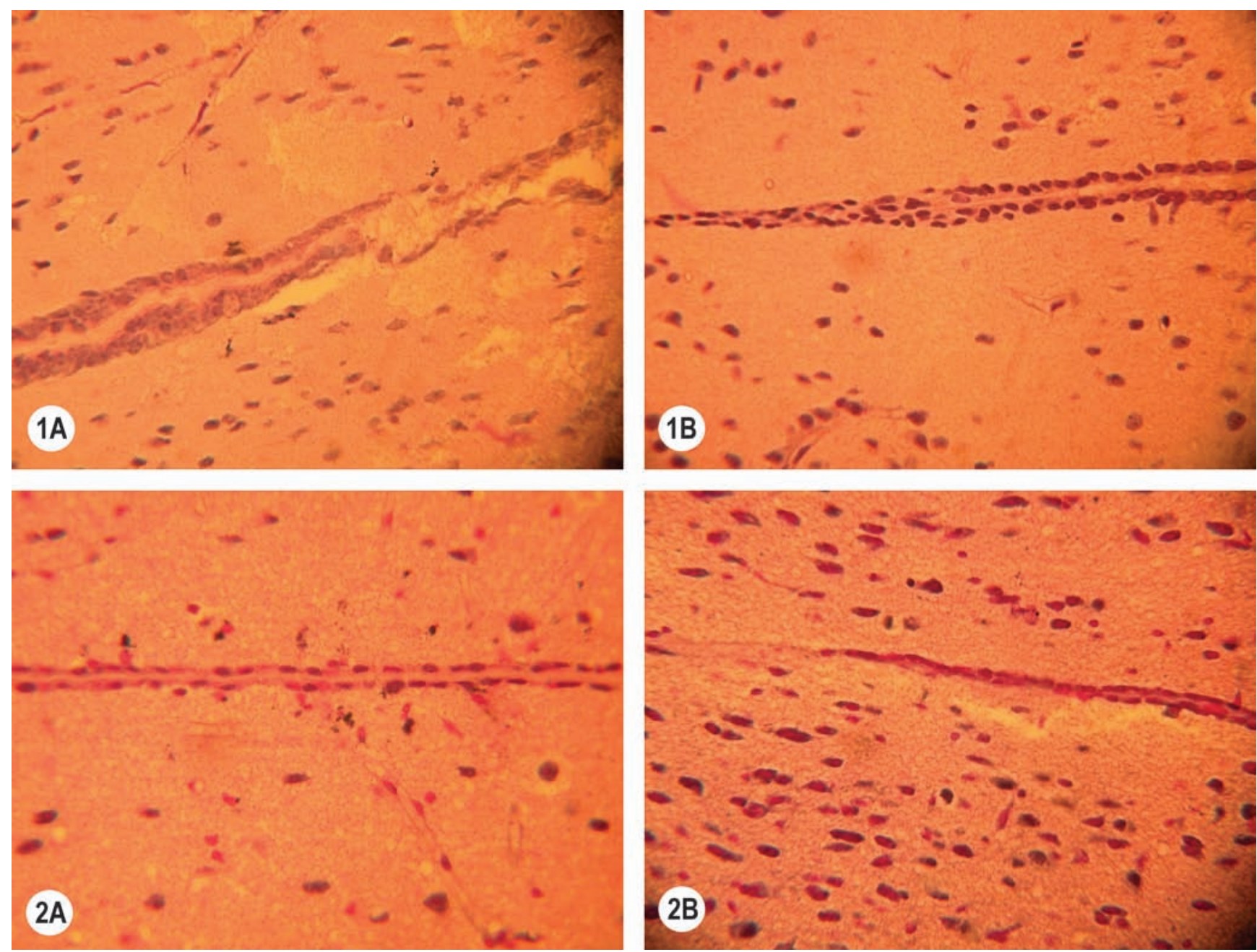

Fig. 1. Fragment of mouse brain third ventricle (III) immersed in control (A) and cyanolytic (B) solution for 5 hours. The glial Gomoripositive material (GGPM) is indicated by arrows. In animal brain after cyanolysis GGPM almost disappeared. Chrome hematoxylinphloxin staining according to Gomori's method (CHP) (magnification $\times 400)$. Fig. 2. A similar fragment of mouse brain immersed in control (A) and cyanolytic (B) solution for 3 hours. After cyanolysis the GGPM also almost totally disappeared. CHP staining (magnification $\times 400)$

induces identical inclusions in cultured, immature astroglia [14]. It was proved also, that cysteamine induced intense cytoplasmic ubiquitin staining, suggesting that activation of the ubiquitin pathway may be an early event in the biogenesis of these astrocytic granules [15]. So, these results support the notation, that the autofluorescent astrocyte inclusions are stress granules which progressively accumulate in the aging periventricular brain.

The Gomori-positivity of the granules may be due to their high sulfur content. The GGPM is positive in the performic acid Alcian blue method. This indicates the presence of protein-bound sulfur [16]. It has been assumed that the sulfur of this material is that of cysteine due to its strong reducing properties [17]. Nevertheless, a possibility remains that it may be that of protein-bound sulfane sulfur [18].

The sulfurtransferases are located in cytosol and mitochondria of the animal cells [7]. The containing sulfur GGPM in periventricular astrocytes appears to be derived from degenerate mitochondria [11].

The aim of this work was to indicate whether most, if not all, of the GGPM is in fact sulfane sulfur.

\section{Materials and methods}

Animals. Twenty eight adult male mice of the CBA strain 6-8 weeks old weighing $\sim 25 \mathrm{~g}$ were used ( 14 for the experiment and 14 controls). The animals were kept under standard laboratory conditions and were fed a standard chow.

Chemicals. Potassium cyanide $(\mathrm{KCN})$ was provided by Merck (Darmstadt, Germany). Potassium hydroxide and other chemicals were obtained from the Polish Chemical Reagent Company (POCh, Gliwice, Poland).

Histology. The animals were killed by cervical dislocation and immediately thereafter the brains were removed and immersed in a $0.1 \mathrm{M}$ potassium cyanide solution, $\mathrm{pH}=9.0$ at room temperature. 

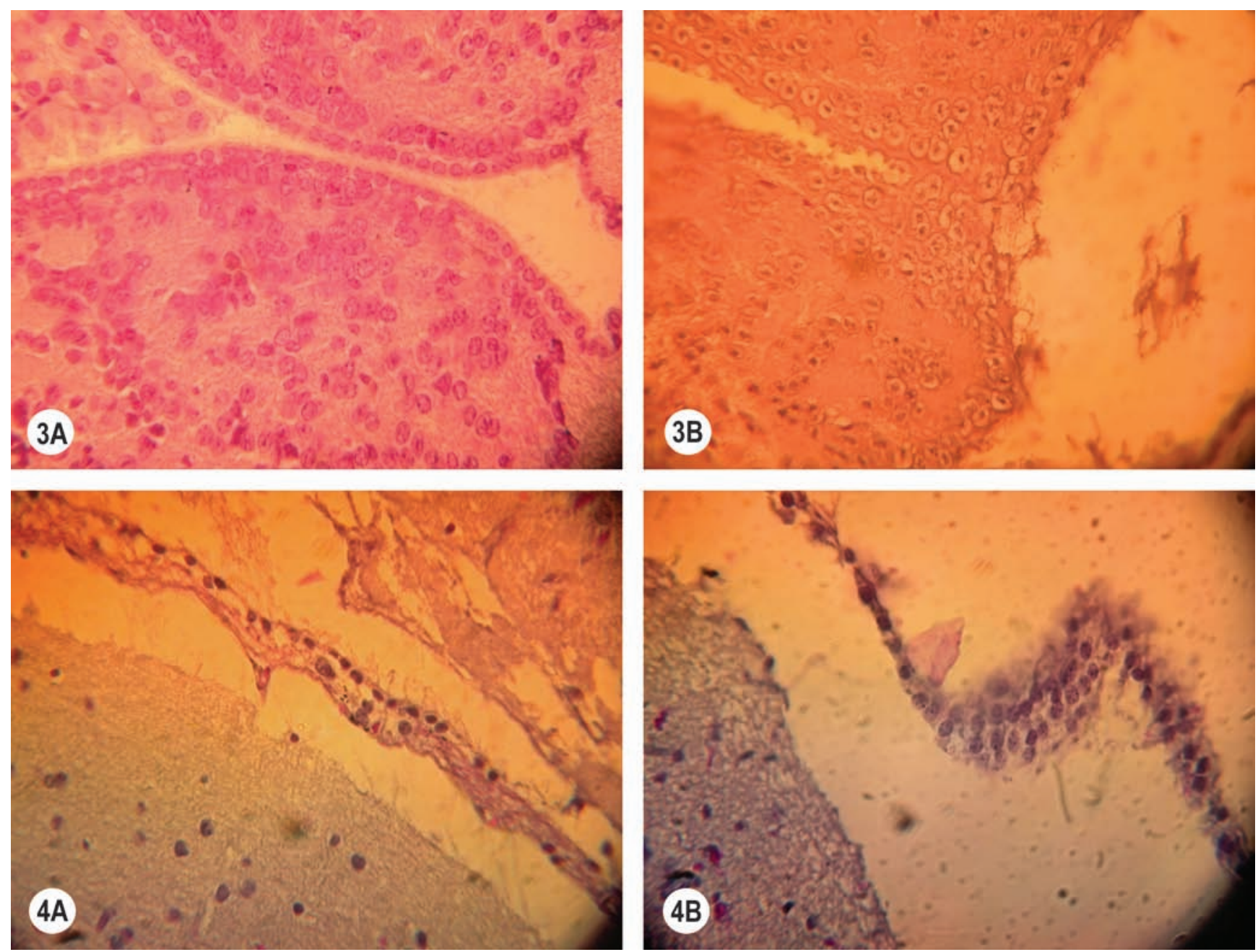

Fig. 3. Fragment of mouse brain showing the subependymal layer (sep) of habenula in control animal (A) and after 30 min of cyanolysis (B). In control mouse subependymal neurons have abundant cytoplasm positively staining with chrome hematoxylin-phloxin, whereas after cyanolysis this stainability disappears. The deeper neurons (n) are also rich in sulfane sulfur. CHP staining (magnification $\times 400$ ). Fig. 4. Fragment of mouse brain showing ependymal lining (ep) of the 4th brain ventricle (IV) in control animal (A) with GGPM (arrows) and after $15 \mathrm{~min}$ in cyanolytic solution (B) with small remnants only of GGPM. CHP staining (magnification $\times 400$ ).

Control brains were immersed in a potassium hydroxide solution, $\mathrm{pH}=9.0$. Thereafter the brains were rinsed in physiological saline and immersed in Bouin's fluid after $15 \mathrm{~min}, 30 \mathrm{~min}, 1 \mathrm{~h}, 3 \mathrm{~h}, 5 \mathrm{~h}, 14 \mathrm{~h}$ or $22 \mathrm{~h}$ of incubation, respectively. Then they were dehydrated in a rising concentration series of ethyl alcohol and paraffin-embedded material was cut serially into $7 \mu \mathrm{m}$ sections and stained with the Gomori's chrome hematoxylin-phloxin method [19].

Ethical issues. All procedures were approved by the Ethic Committee for the Animal Research in Krakow (no. 40/2007/19.04).

\section{Results and discussion}

Figure 1A shows fragment of the third ventricle of control brain, which was immersed in potassium hydroxide solution for $5 \mathrm{~h}$. This control brain contains numerous glial Gomori-positive granules (indicated in Fig. 1A by arrows). In Fig. 1B fragment of the third brain ventricle after $5 \mathrm{~h}$ of cyanolysis is presented and the GGPM has almost completely disappeared, which, indicates, that this time period is inefficient for whole cyanolysis or that a small fragment of some GGPM particles contains sulfur different from sulfane sulfur. This may be the sulfur of cysteine or cystine. A similar fragment of the brain following $3 \mathrm{~h}$ cyanolysis is also almost totally devoid of the GGPM (Fig. 2B) whereas the control fragment shows numerous Gomori-positive glial cells (Fig. 2A).

Figures $3 \mathrm{~A}$ and $3 \mathrm{~B}$ present the subependymal layer of the habenula after $30 \mathrm{~min}$ of cyanolysis. Superficial, subependymal cells of the habenula have abundant cytoplasm positively staining with Gomori's chrome hematoxylin-phloxin (Fig. 3A), whereas this stainability disappears after cyanolysis (Fig. 3B). This finding indicates that these cells possibly also are rich in sulfane sulfur. The deeper cells are neurons (Fig. 3A and $\mathrm{B})$ and this finding shows that some neurons of the habenula are rich in sulfane sulfur. 
Figure 4B shows a fragment of ependymal lining of the 4th brain ventricle after 15 min of cyanolysis. Very small remnants of GGPM can be observed in some specimens. In a control animal (Fig. 4A) GGPM can be found. Small traces of GGPM remained through the later periods of cyanolysis until $22 \mathrm{~h}$.

The cyanolysability of GGPM indicates that its stainable component is sulfane sulfur. GGPM is relatively stable, not being degraded during fixation of the tissue in Bouin's fluid and oxidation of the sections with permanganate as is required to obtain the stainability with chrome hematoxylin-phloxin Gomori's method. As regards to the role of GGPM, it may serve as an antioxidant and scavenge free radicals. Protective properties of sulfane sulfur is connected with the possibility of scavenging free radicals as well as with the ability to increase of antioxidative enzymes activity. Persulfides are more efficient hydrogen donors in comparison with sulfydryl groups, and being persulfide anions, they are more reactive electron donors, which makes them remarkably effective antioxidants [20].

A protective role for the Gomori-positive glia has been proposed repeatedly since it has been shown to accumulate silver nitrate injected to rats [21]. In vivo, numbers of astrocytic granules increase as a function of advancing age, in response to chronic estrogen stimulation, and following X-radiation [17] and after treatment with some xenobiotics [16]. In vitro, these cells accumulate with increasing time in culture and following exposure to the sulfhydryl agent, cysteamine [11]. These cells may contribute to the pathogenesis of several neurologic disorders, especially potentiation of parkinsonism and other free radical-related neurodegenerations [11]. However, injection of potassium cyanide causes significant decrease in number of GGPM containing glial cells [18], what suggests the role of GGPM in cyanide detoxification. Results in vivo studies of Buzaleh et al. [22,23] revealed, that upon chronic cyanide treatment, sulfane sulfur level and rhodanese activity were elevated only in blood and in the heart. This implies that during cyanide treatment, sulfur is released from GGPM into blood, and in this way can participate in cyanide detoxification. Our previous studies indicated that the number of cells containing GGPM increased under the influence of sulfane sulfur donor (diallyl disulfide) and dropped after administration of cyanide [18]. These findings together with results presented in this paper suggest, that GGPM observed in periventricular astrocytes of mice and other mammals can be a source and a store of sulfane sulfur in mammals.

GGPM may also be a "sink" for free cysteine that has to be removed from brain tissue. Free L-cysteine appears in the brain tissue as a result of neuronal activity; it is released from a neuronal compartment and might be involved in neurotransmission [24] but, at the same time, after systemic administration to immature rodents, L-cysteine destroys neurons in the cerebral cortex, hippocampus, thalamus, and striatum, thus being a bicarbonate-sensitive endogenous excitotoxin [25].

In summary, cyanolysis experiments presented in this paper, have confirmed previous hypothesis, that the Gomori-positive material present in periventricular astrocytes is a sulfane sulfur, possibly in the form of protein bound cysteine persulfide.

\section{References}

[ 1] Bagley PJ, Stipanuk MH. Rats fed a low protein diet supplemented with sulfur amino acids have increased cysteine dioxygenase activity and increased taurine production in hepatocytes. J Nutr. 1995;125:933-940.

[2] Iciek M, Włodek L. Biosynthesis and biological properties of compounds containing highly reactive, reduced sulfane sulfur. Pol J Pharmacol. 2001;53:215-225.

[3] Sörbo B. Sulfite and complex-bound cyanide as sulfur acceptors for rhodanese. Acta Chem Scand. 1957;11:628-633.

[ 4] Wood JL. Sulfane sulfur. Methods Enzymol. 1987;143:25-29.

[5] Toohey JL. Sulfane sulfur in biological systems: a possible regulatory role. Biochem J. 1989;264:625-632.

[6] Nandi DL, Westley J. Reduced thioredoxin as a sulfur acceptor substrate for rhodanese. Int $J$ Biochem Cell Biol. 1998;30:973-977.

[7] Koj A, Frendo J, Wojtczak L. Subcellular distribution and intramitochondrial localization of three sulfurtransferases in rat liver. FEBS Lett. 1975;57:42-46.

[ 8] Nagahara N, Ito T, Kitamura H, Nishino T. Tissue and subcellular distribution of mercaptopyruvate sulfurtransferase in the rat: confocal laser fluorescence and immunoelectron microscopic studies combined with biochemical analysis. Histochem Cell Biol. 1998;110:243-250.

[9] Srebro Z. A comparative and experimental study of the Gomori positive glia. Folia Biol. (Kraków), 1966;17:177-192.

[10] Srebro Z, Lach H. The ultrastructure of the periventricular glia in the brains of rats and mice. Folia Biol. (Kraków), 1987;35:131-136.

[11] Schipper HM. Gomori-positive astrocytes: biological properties and implications for neurologic and neuroendocrine disorders. Glia. 1991;4:365-377.

[12] Schipper HM, Cisse S. Mitochondrial constituents of corpora amylacea and autofluorescent astrocytic inclusions in senescent human brain. Glia. 1995;14:55-64.

[13] Schipper HM, Cisse S, Walton PA. Colocalization of organelle-specific proteins to autofluorescent astrocyte granules by laser scanning confocal microscopy. Exp Cell Res. 1993;207:62-67.

[14] Cisse S, Schipper HM. Isolation of pseudoperoxidase-positive astrocyte granules from intact rat brain and cysteaminetreated neuroglial culture. Brain Res. 1993;615:141-146.

[15] Mydlarski MB, Schipper HM. Stress protein co-localization to autofluorescent astrocytic inclusions in situ and in cysteamine-treated glial cultures. Brain Res. 1993;627:113121.

[16] Srebro Z, Szirmai E. The neurosecretory system and the periventricular glia of rats treated with salicyl-quinine-lithium. Gaz Int Med Chir Rome. 1971;76:1218-1227.

[17] Srebro Z. X-ray induced increase in number of cysteine-rich periventricular glial cells in the rat brain. Experientia. 1971;27:945-947. 
[18] Iciek M, Bilska A, Książek L, Srebro Z, Włodek L. Allyl sulfide as donor and cyanide as acceptor of sulfane sulfur in the mouse tissues. Pharmacol Rep. 2005;57:212-218.

[19] Pearse AG. Histochemistry, Theoretical and Applied. London: Churchill; 1960.

[20] Everett SA, Folkes LK, Wardman P. Free radical repair by a novel perthiol: reversible hydrogen transfer and perthiyl radical formation. Free Radic Res Commun. 1994;20:387-400.

[21] Wislocki GB, Leduc EH. The cytology of the subcommissural organ, Reissner's fiber, periventricular glial cells and posterior collicular recess of the rat's brain. J Comp Neurol. 1954; 101:283-309.

[22] Buzaleh AM, Vázqez ES, Battle AM. Cyanide intoxicationI. An oral chronic animal model. Gen Pharmacol. 1989;20: 323-327.
[23] Buzaleh AM, Vázqez ES, Battle AM. Cyanide intoxication-II. The effects of systematic cyanide challenge on indicative toxicity parameters. Comp Biochem Physiol C. 1990;96:177180.

[24] Olney JW, Ho OL, Rhee V. Cytotoxic effects of acidic and sulfur-containing amino acids on the infant mouse central nervous system. Exp Brain Res. 1971;14:61-76.

[25] Olney JW, Zorumski C, Price MT, Labruyere J. L-cysteine, a bicarbonate-sensitive endogenous excitotoxin. Science. 1990;248:596-599. 